\title{
AUTONOMOUS NAVIGATION OF A MOBILE ROBOT USING CUSTOM-DESIGNED QUALITATIVE REASONING VLSI CHIPS AND BOARDS*
}

\author{
François G. Pin, Hiroyuki Watanabe, ${ }^{*}$ Jim Symon, ${ }^{*}$ and Robert S. Pattay \\ Center for Engineering Systems Advanced Research \\ Oak Ridge National Laboratory \\ P.O. Box 2008 \\ Oak Ridge, TN 37831-6364, U.S.A.
}
Contact: Dr. François G. Pin, Oak Ridge National Laboratory, P.O. Box 2008, Building 6025, MS-6364, Oak Ridge, TN 37831-6364, U.S.A., Telephone: (615)574-6130, Fax: (615)574-7860, Email: pin@stc10.ctd.ornl.gov

Submitted to: The 1992 IEEE International Conference on Robotics and Automation, Nice, France, May 10-15, 1992

\footnotetext{
* Research sponsored by the Office of Engineering Research Program, Basic Energy Sciences, of the U.S. Department of Energy, under contract no. DE-AC05-840R21400 with Martin Marietta Energy Systems, Inc.

* Department of Computer Science, University of North Carolina at Chapel Hill, Chapel Hill, NC 27599
}

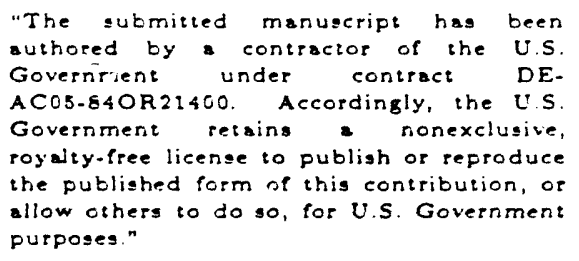
the U.S. allow others to do so, for U.S. Government putposes." 


\title{
AUTONOMOUS NAVIGATION OF A MOBILE ROBCT USING CUSTOM-DESIGNED QUALITATIVE REASONING VLSI CHIPS AND BOARDS
}

\author{
François G. Pin, Hiroyuki Watanabe," Jim Symon," and Robert S. Pattay \\ Center for Engineering Systems Advanced Research \\ Oak Ridge National Laboratory \\ P.O. Box 2008 \\ Oak Ridge, TN 37831-6364, U.S.A.
}

\begin{abstract}
Two types of computer boards including custom-designed VLSI chips have been developed to add a qualitative reasoning capability to the real-time control of autonomous mobile robots. The design and operation of these boards are first described and an example of their use for the autonomous navigation of a mobile robot is presented. The development of qualitative reasoning schemes emulating human-like navigation in a-priori unknown environments is discussed. The efficiency of such schemes, which can consist of as little as a dozen qualitative rules, is illustrated in experiments involving an autonomous mobile robot navigating on the basis of very sparse and inaccurate sensor data.
\end{abstract}

\section{INTRODUCTION}

One of the greatest chailenges in the motion planning and control of autonomous mobile robots in a-priori unknown or dynamic environments is to provide the reasoning modules with methods for handling and/or coping with the many imprecisions, inaccuracies, and uncertainties present in the system. These typically arise from three major sources: (1) errors in the sensor data (there are no perfect sensor systems) which lead to inaccuracies and uncertainties in the representation of the environment, the robot's estimated position, etc., (2) imprecisions or lack of knowledge in our understanding of the system, i.e., our inability to generate complete and exact (crisp) mathematical and/or numerical descriptions of all the phenomena contributing to the system's and environment's behavior, and (3) approximations and imprecisions in the information processing schemes (e.g., discretization, numerical truncation, convergence thresholds, etc.) that are used to generate decisions or control output signals.

* Department of Computer Science, University of North Carolina at Chapel Hill, Chapel Hill, NC 27599 
Qualitative reasoning (also termed approximate reasoning) refers to a set of methodulogies which have been developed to provide alternative solutions methods for decision-making problems when the uncertainties can not be fully engineered away (e.g., there are limits on maximum sensor precision, predictability of the environment, etc.). The general approach underlying these methodologies consist in capturing some aspects of the reasoning methods typically exhibited by humans when controlling systems, by implicitly incorporating uncertainties in the information gathering and reasoning processes, rather than attempting to explicitly determine and propagate them through numerical calculations or representations. Several approximate reasoning theories and associated mathematical algebra have been developed over the past two decades [1], the most commonly used today for applications to control systems being Zadeh's Theory of Fuzzy Sets [2]-[5]. This theory is at the basis of very successful implementations varying from control of subway cars, cement kilns, washing machines, still and video cameras, inverted pendulums, to painting processes and color image reconstruction, to even ping-pong playing roiots [6]-[12].

One of the important factors which have prevented the wide-spread utilization of approximate reasoning in real-time systems has been the unavailability of computer hardware allowing processing and inferencing directly in terms of approximate or linguistic, or "fuzzy" variables (e.g., far, fast, slow, left, faster, etc.) and approximate rules (e.g., if obstacle is close, then go slower; if temperature is high and pressure is increasing, then decrease power a lot, etc.). Prospective implementations thus had to rely on simulation of the approximate reasoning schemes on conventional hardware and computers based on "crisp" (numerical) processing, with a resulting significant penalty in speed of operation, prohibiting applications in most "hard real-time" systems.

In cooperation with Micro Electronics, Inc., unique computer boards have recently been dereloped using custom-designed VLSI chips [13],[14] which can be programmed to directly communicate and interface in terms of qualitative variables and rules. Additionally; the boards' architecture is reconfigurable on-line to allow several levels of reasoning (meta level, non monotonic, etc.) and to allow full inferences with up to 350 rules and 28 input channels to take place in $30 \mu \mathrm{sec}$, i.e., at a rate of $30,000 \mathrm{~Hz}$ (at least two orders of magnitude faster than video frame rate). This paper provides an overview of the design and opcration of these boards and discusses their first implementation in the development of approximate reasoning methodologies and schemes for CESAR's series of HERMIES (Hostile Environments Robotic Machine Intelligence Experiments) test-bed robots. 


\section{QUALITATIVE REASONING ON A VLSI CHIP}

The qualitative reasoning methodology utilized for the VLSI implementation is inspired from the Theory of Fuzzy Sets, in which the functicns $\mu_{X}(x)$ defining the membership of an element $x$ to a subset $X$ of a universe of discourse $U$ can take any value in the interval $[0,1]$, rather than only the discrete $\{0,1\}$ values ( 0 for does not belong, 1 for belongs) used in conventional (crisp) Set Theory. The function $\mu_{X}(x)$ thus defines the degree of membership of the element $x$ in $X$. Such a subset $X$ of $U$ is termed a qualitative (or approximate, conceptual, or fuzzy) variable for reasoning on the universe of discourse $U$.

For the current VLSI implementation, reasoning is embodied in programmable "production rules" operating on four sets of qualitative input variables and two sets of output qualitative variables, as in

IF ( $A$ is $A_{1}$ and $B$ is $B_{1}$ and $C$ is $C_{1}$ and $D$ is $\left.D_{1}\right)$ THEN ( $E$ is $E_{1}$ and $F$ is $F_{1}$ ),

where $A_{1}, B_{1}, \ldots F_{1}$ are qualitative rariables whose representative membersli-1, functions define the rule, and $A, B, C \ldots F$ are the time-rarying qualitative input and output variables analogous to memory elements in conventional production systems.

With the above representation, the Fuzzy Set Theoretic Operations can be directly applied to the qualitative variables on their universe of discourse: given two subsets $A$ and $B$ of $U$,

$$
\begin{aligned}
& \mu_{A \cap B}(x)=\min \left(\mu_{A}(x), \mu_{B}(x)\right) \\
& \mu_{A U B}(x)=\max \left(\mu_{A}(x), \mu_{B}(x)\right)
\end{aligned}
$$

The laws of logical inferences including modus ponens, cartesian product, projection and compositional inferences (e.g., see [3] and [4] for detailed description of these laws of inferencing) can also be applied to multivariable systems. In particular, the extension principle [3],[4] is used in the mapping between a set $A$ of the input universe of discourse $U$ and its extension through $F$ to the output universe of discourse $V$, as:

$$
\mu_{F(A)}(v)=\operatorname{Sup}_{u} \mu_{.4}(u)
$$

where $v=F(u), u \epsilon U, v \epsilon V$.

For their VLSI interpretation, each qualitative variable is represented by its membership function discretized over a $(64 \times 16)$ array of $(x, \mu(x))$ values. Equations (1), (2), (3), and (4) can thus be easily implemented using series of min. and max. gates as shown in Fig. 1 for one rule. Figure 2 schematically represents an inference with two rules 
of the form IF ( $A$ is $A^{1}$ and $B$ is $B^{1}$ ) THEN ( $E$ is $E^{1}$ ) operating on two input $A$ and $B$ and producing a composite membership function for $E$.

IP.Pars

TUEN.Pars

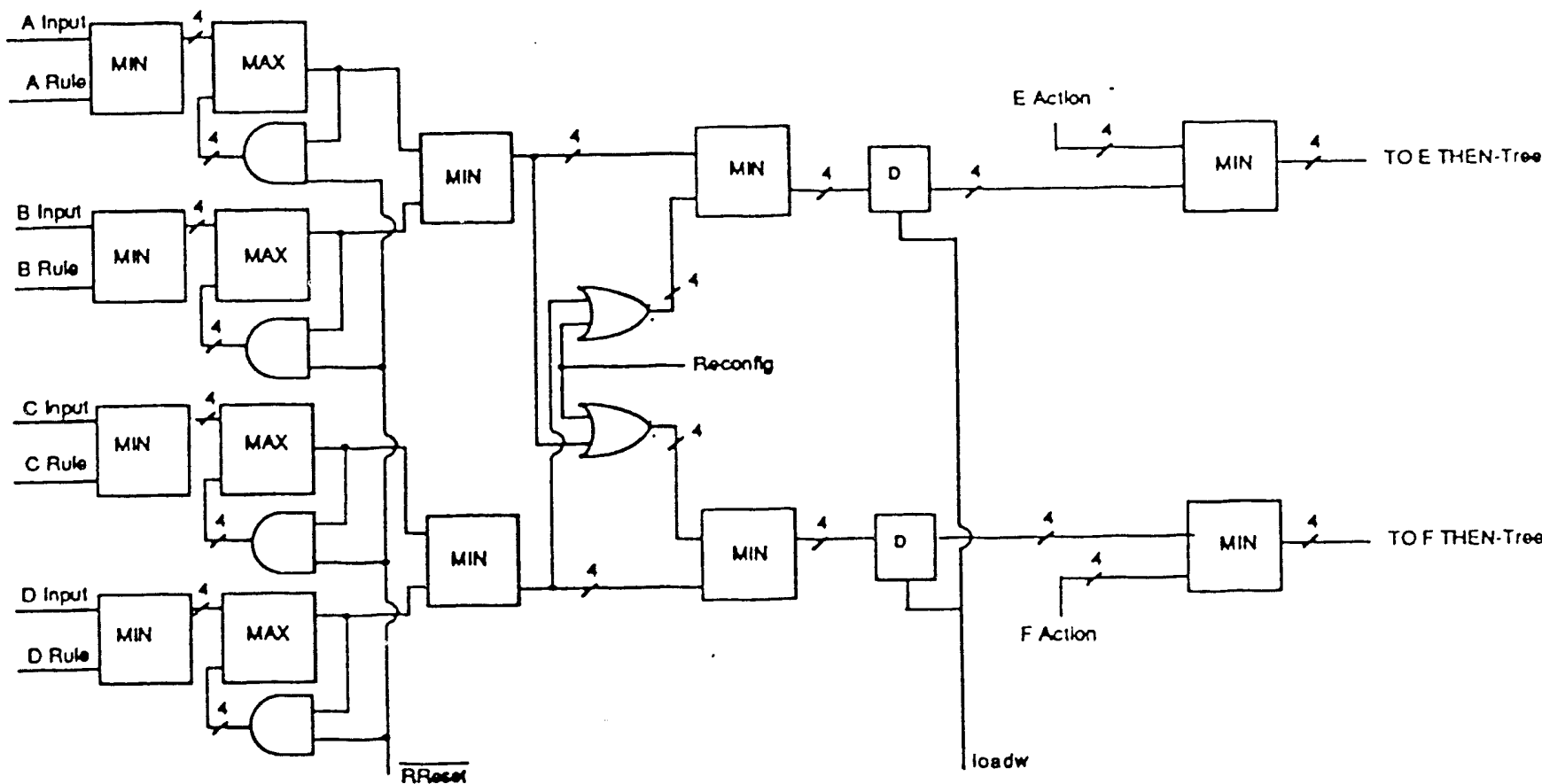

Fig. 1. Data path for rule execution.
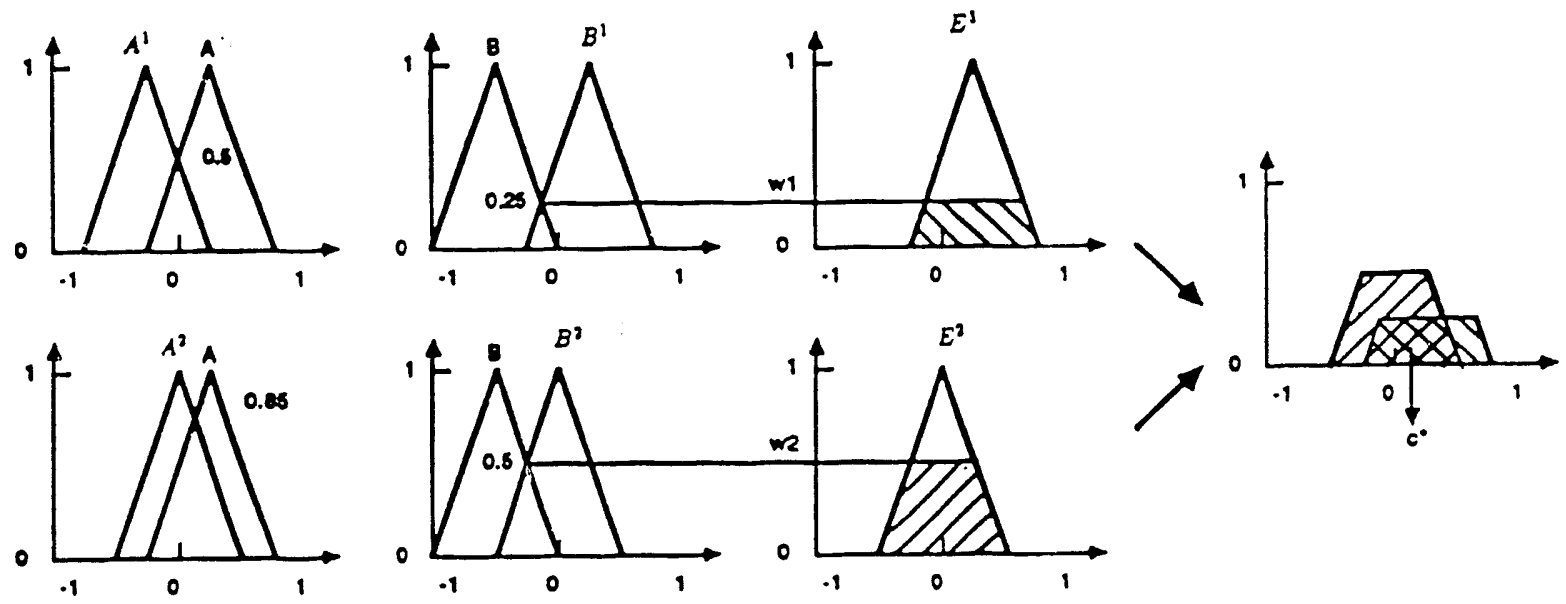

Fig. 2. Schematic of a qualitative inference using two rules operating on two input and one output channels. 
Because conventional sensors typically provide data in "crisp" form, i.e., they provide a single number and the uncertainty on their measurement is ignored, it is desirable to add this uncertainty on the measurement, effectively mapping it to a qualitative variable, prior to processing through approximate reasoning. This step (which has been termed fuzzification) is of course not necessary if the data is already in the form of a qualitative variable, such as in interchip communications, and therefore has been implemented as a programmable optional data path on the VLSI chip. Similarly, an optional defuzzing step which calculates the "center of weight" of the outpui composite membership function (see Fig. 2), can be used to send "crisp" data to conventional actuators if these are used in the process control hardware as depicted on Fig. 3. To provide added flexibility; the chip architecture is reconfigurable, allowing either 50 rules operating on four input and two output channels or 100 rules operating on two input and one output channels. Since all rules are processed in parallel, the speed of operation of the chip is independent of the configuration or the number of rules involved in the inferencing, and reaches 30,000 FFIPS (Full Fuzzy Inferences Per Second). In other words, full qualitative data processing and inferencing schemes can take place at $30 \mathrm{KHz}$, (i.e., at least two orders of magnitude faster than the sampling rate of typical sensors) making feasible the control of very fast systems or motions, such as those involved in reflex behaviors based on very approximate or uncertain informations. User

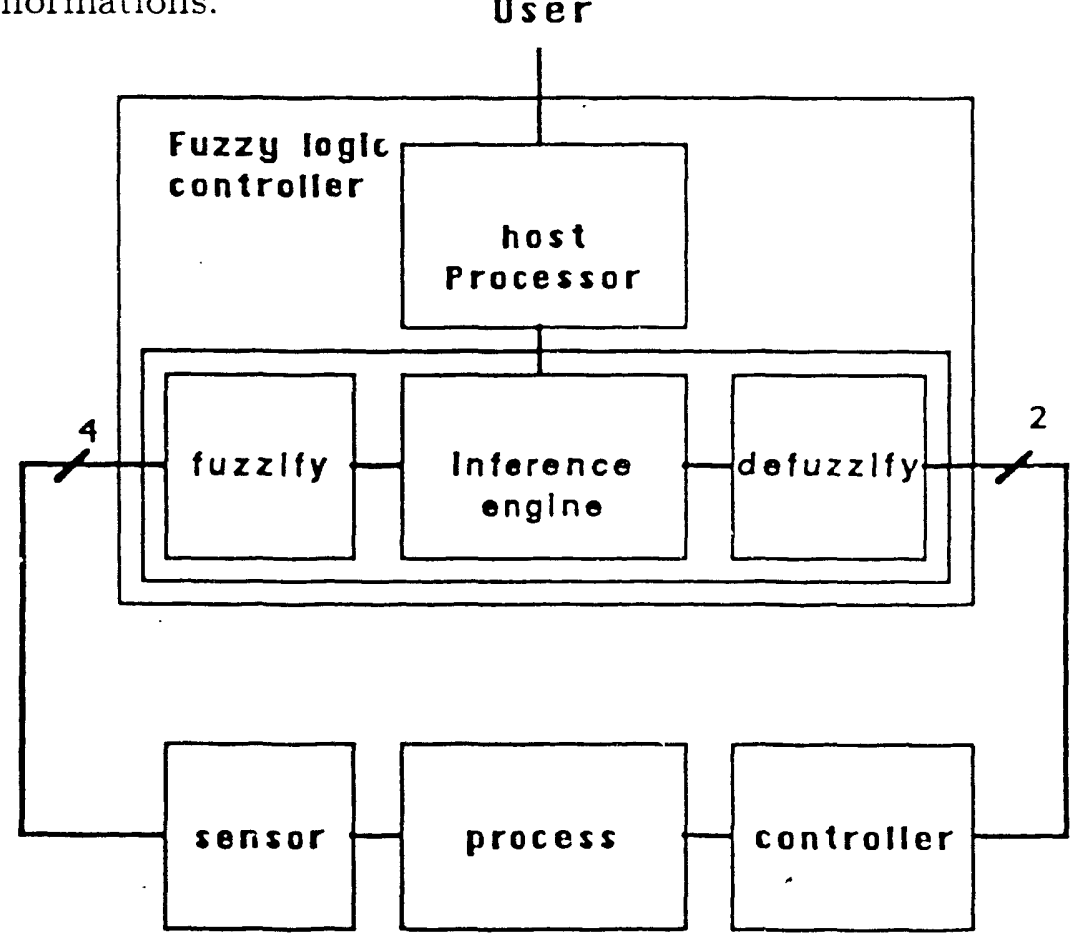

Fig. 3. Schematic of a typical qualitative control system for a real-time process. 
Two types of VMEbus-compatible printed boards and associated software were developed to allow interfacing of the chips with sensors and actuator data channels for application to "intelligent machines" and in particular autonomous mobile robots. The first type of board includes one chip and is therefore limited to inferencing involving only 4 input and 2 output channels. The second type of board includes 7 chips and some multiplexer circuits which allow on-line reconfiguration of the input, output and interchip communication paths. This provides the capability to implement qualitative reasoning schemes with up to 350 rules and 28 input channels (with all chips in parallel), multi-level reasoning schemes (e.g., 4 chips in a first layer feeding into 2 chips in the second layer feeding into 1 chip in the third layer), or non-monotonic reasoning (e.g., with feedback of the output of some of the downstream chips into the input of some of the upstream chips, in a series or "cascade" of chips). The speed of operation of each layer of parallel chips remains the same than on the single chip board, with the multi-layer configurations reaching rates in the $\mathrm{KHz}$ order of magnitude.

\section{TEST IMPLEMENTATION FOR MOBILE ROBOTS NAVIGATION}

The problem of autonomous mobile robots navigating in a-priori unknown and unpredictable environments was selected for initial testing of the qualitative reasoning systems because its characteristics rank very high on the list of criteria that typically indicate suitability of a reasoning scheme for representation and implementation using qualitative logic: tile input to the control system, particularly when provided by sonar range finders and odometry wheel encoders, is extremely inaccurate, sparse, uncertain and/or unreliable; there exist no complete mathematical and/or numerical representation of the behavior termed navigation, although, as demonstrated by humans, a logic for this behavior exists which can typically be represented and successfully processed in terms of linguistic variables; by its given nature the behavior of the environment is unpredictable, leading to large uncertainties in its representation; the approximations involved in the numerical representation of the system and its environment (e.g., geometric representations, map discretization in grid, etc.) are significant.

The single chip board and a recently designed omnidirectional mobile platform [15],[16] were used for these initial experiments. Because of the limitations to 4 input channels using this board, data from only 3 frontal sonars were used for perception of the environment, while the fourth channel produced information on angular direction to the given navigation goal based on odometry sensor data. The output channels provided translational speed and steering velocity commands to be sent to the motor controls. No 
a-priori environmental data or inaps were input to the system, nor were any generated during motion, and in this sense, the initial investigations focussed on reactive navigation. The development and testing of the first series of qualitative rule-bases led to empirical findings providing significant insights for efficient implementation of qualitative reasoning schemes in autonomous robots:

- Modularity and consistency of the rule-base can best be achieved through decomposition of the decision-making scheme into elemental and independent "behaviors."

- Independence of these elemental behaviors is assured if each can be formulated as a direct mapping between a subset of the input and a subset of the output, with no redundancy in the qualitative values spanned by the input variables of various behariors.

- Independent behaviors can be singly developed and tested, and their independence experimentally verified prior to merging with other behaviors.

- Once developed, tested and rerified, each behavior can be assigned a normalized "weight" (in $[0,1]$ ) corresponding to its relative importance with respect to other behaviors with which it is to be merged, (e.g., safety from obstacle rs. speed of operation, etc.). The weighting is implemented by a direct scaling of the membership functions of either the input or the output variables.

- Merging of the behaviors is handled directly and continuously through the laws of combinatorial inferencing, therefore providing a formal resolution to one of the major problems with which the "behaviorist" community (e.g., see [17] and [18] and references therein) has struggled: the real-time selection and/or conflict resolution in multibehavior systems.

Building upon the experience and empirical results gained during the development of the first series of rule-bases, a new rule-base conforming with the observations listed above was developed for the single chip board and the CESAR's omnidirectional platform pictured in Fig. 4. The photograph in the figure shows the ring of accoustic range sensors at the edge of the platform deck (only frontal sensors are used) and the disk drive unit, the battery pack (rear right) and the seven-slot VME-bus (rear left) which hosts the qualitative inferencing board. The control system of the platform (detailed in [15] and [16]) includes a velocity loop servoing at $100 \mathrm{~Hz}$ on the commanded translational and rotational velocities, which will be hereafter referred to as speed control and turn control, respectively. Thus, behaviors corresponding to speed control (S.C.) and turn control (T.C.) as functions of goal orientation (G.O.) and obstacle proximity (O.P.) where developed as follows: 


$\begin{array}{rr}\text { G.O. } \longrightarrow \text { S.C. } & (1 \text { rule }) \\ \text { O.P. } \longrightarrow \text { S.C. } & (4 \text { rules }) \\ \text { G.O. } \longrightarrow \text { T.C. } & \text { (2 rules) } \\ \text { "far" O.P. } \longrightarrow \text { T.C. } & (2 \text { rules) } \\ \text { "near" O.P. } \longrightarrow \text { T.C. } & (2 \text { rules) } \\ \text { "very close" O.P. } \longrightarrow \text { T.C. } & \text { (3 rules) }\end{array}$

where the three latter behaviors embody the fact that different navigation behaviors are utilized deperding on whether all obstacles are still "far," "near," or "very close," thus reflecting differences in safety concerns (i.e., priority of the behavior) implemented using different weights. For each sampling period and decision, several behaviors are typically triggered and merged through the Fuzzy Set Theoretic laws of Combinatorial Inferencing, resulting in a smooth and continuous sensor-based navigation control.

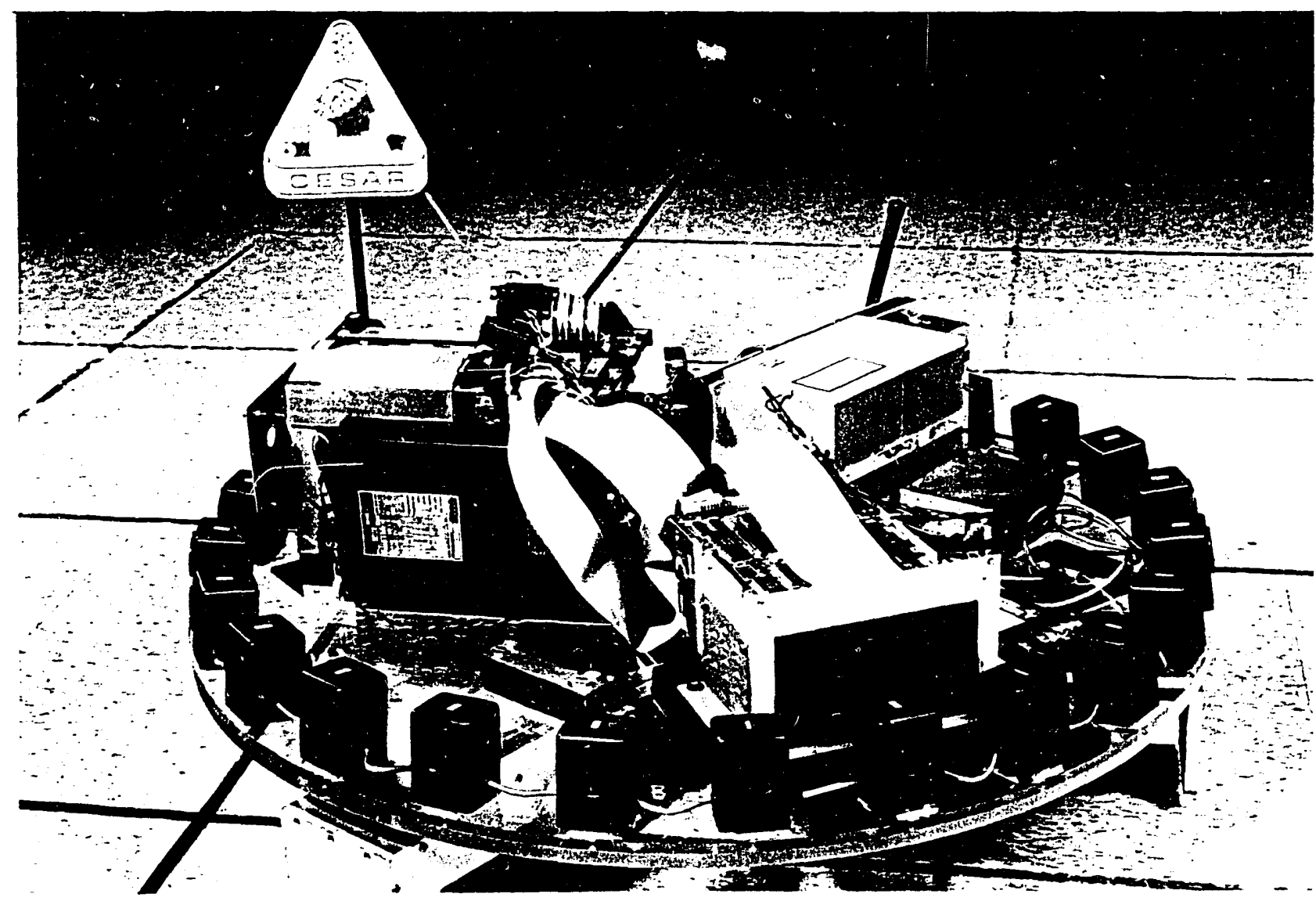

Fig. 4. The CESAR omnidirectional robotic platform prototype.

The rules for TC and SC as a function of GO express the rery intuitive fact that if the goal is to the left (respectively right), then a small increment of turn to the left (respectively right) needs to be made during the loop rate cycle; and when the direction of the goal increases from $0^{\circ}$ (front) to $\pm 180^{\circ}$, then the speed is correspondingly decreased. The rules for $\mathrm{SC}$ as a function of $\mathrm{OP}$ express that when the distances to any obstacles (i.e., the sensor 
returns in all three directions) are increasing, then the speed can be increased toward its maximum value (one rule), while when distance to an obstacle (sonar return) in any of the three sonar directions (thus, three rules) decreases toward a safety threshold (here selected as $30 \mathrm{~cm}$ ), then the speed needs to be decreased, down to zero at or below that threshold.

Once these speed control and goal tracking behaviors were designed, they were merged and tested in environments with no obstacles. Since the chip used for this initial implementation allowed only four input channels, no information related to distance to the goal could be provided to the qualitative reasoning scheme in order to make the robot stop when reaching the goal. This was easily remedied in these tests by using the odometry data in the master program to stop both the reasoning scheme and the robot when it approached to within a given radius $(2.5 \mathrm{~cm})$ of the goal. In future implementations using the seven-chip board allowing up to 28 input channels, the distance to the goal could be input to the qualitative inference scheme and the stopping at the goal could be simply implemented as an additional behavior in the reasoning scheme.

Once these behaviors were tested, the rules for the TC as a function of OP behaviors were developed. When all sonar returns are "far" (turther away than $2 \mathrm{~m}$ ), the turn should be away from the closest obstacle. However, the weight on that behavior must be less than that for the $\mathrm{TC}$ as a function of GO, to ensure that when it is far away from any obstacles, the robot's priority is still to move in the general direction of the goal. When at least one of the sonar returns is "near" (between $30 \mathrm{~cm}$ and $2 \mathrm{~m}$ ), the turn is away from the obstacle, increasing in magnitude with decreasing distance, such that at the lowest distance of $30 \mathrm{~cm}$, this obstacle avoidance behavior has more weight on TC than the goal tracking behavior. Finally when any sonar return is less than $30 \mathrm{~cm}$ (the robot is stopped as required by the behavior on $\mathrm{SC}$ as a function of $\mathrm{OP}$ ), the turn is always to the right. Note that setting the turn away from the closest obstacle in this latter behavior would often result in the dead-lock situations in which the robot reaches a limit cycle, and continuously oscillates between two oricntations. This type of situation constitutes one of the very serious drawbacks of the rective navigation methods using potential field techniques, and has been alleviated here using the TC as a function of "very close" OP behavior. Also note that this behavior allows the robot to travel to the end of dead-end corridors, turn around, and backtrack to a more open area, a situation which would lead to a (local minimum) dead-end point in potential field techniques.

Figures 5 and 6 show plots of sample runs made with the robot to illustrate the overall reactive navigation using the qualitative inferencing scheme and, in particular, the two characteristics just discussed. In the figures, the lightly shaded areas represent the obstacles which were placed in the room, while the path of the robot is illustrated using the dark succession of circles. In Fig. 5, the robot initially starts toward the goal, encounters the wall, moves along the wall, passes the point directly opposite to the goal on the 
perpendicular to the wall (at which a dead-lock would be encountered using potential field techniques), and continues until it reaches the end of the wall where it can turn to reach the goal. In Fig. 6, the robot starts toward the goal and, when facing obstacle A head-on, moves in the opening on its right which is closest to the goal direction. When reaching the end of this blocked corridor, the robot turns around (using the TC as a function of "very close" OP behavior), exit the corridor, turns in a direction closest to the goal direction, avoids the small obstacles and then mores to the goal.

\section{SUMMARY AND CONCLUDING REMARKS}

Autonomous robot control in a-priori unknown, liriprediciable, and dymamic environments requires many calculational and reasoning schemes to operate on the basis of very imprecise, incomplete, sparse or unreliable data, knowledge or information. In such systems, for which engineering all the uncertainties away from the hardware is not currently fully feasible, approximate reasoning may provide an alternative to the complexity and computer requirements of conventional uncertainty analysis and propagation techniques.

Two types of computer boards including custom-designed VLSI chips have been developed to investigate the implementation and real-time use of approximate reasoning in autonomous robotic systems. The methodologies embodied on the VLSI hardware utilize the Fuzzy Set Theoretic operations to implement a production rule type of inferencing on input and output variables that can directly be specified as qualitative variables through membership functions. All rules on a chip are processed in parallel, allowing full inferences to take place in about $30 \mu \mathrm{sec}$. This speed of operation makes real-time reasoning feasible at rates much faster than sensor data acquisition, therefore, making control of "reflex-type" of motions envisionable.

One of the qualitative inferencing boards, incorporating one chip with four input channels and two output channels, was installed on a test-bed platform to investigate the use of qualitative reasoning schemes for the autonomous navigation of a mobile robot in a-priori unknown environments on the basis of sparse and imprecise data. Experiments in which the robot uses only three accoustic range (sonar) sensors have demonstrated the feasibility of basic reactive navigation with a scheme including six elemental behaviors represented in fourteen-qualitative-rules. The approach using superposition of behaviors allows to progressively merge additional behaviors into the scheme to resolve any specific additional situation which may be encountered in particular environments of increasing complexity. Our ongoing work focusses on this area, utilizing the recently completed multi-chip board (which allows up to 28 inputs and 14 outputs) to investigate schemes with additional input variables and greater numbers of behaviors, for which we were limited in this first series of expei:ments by the four-input-only restriction of the single-chip board. 


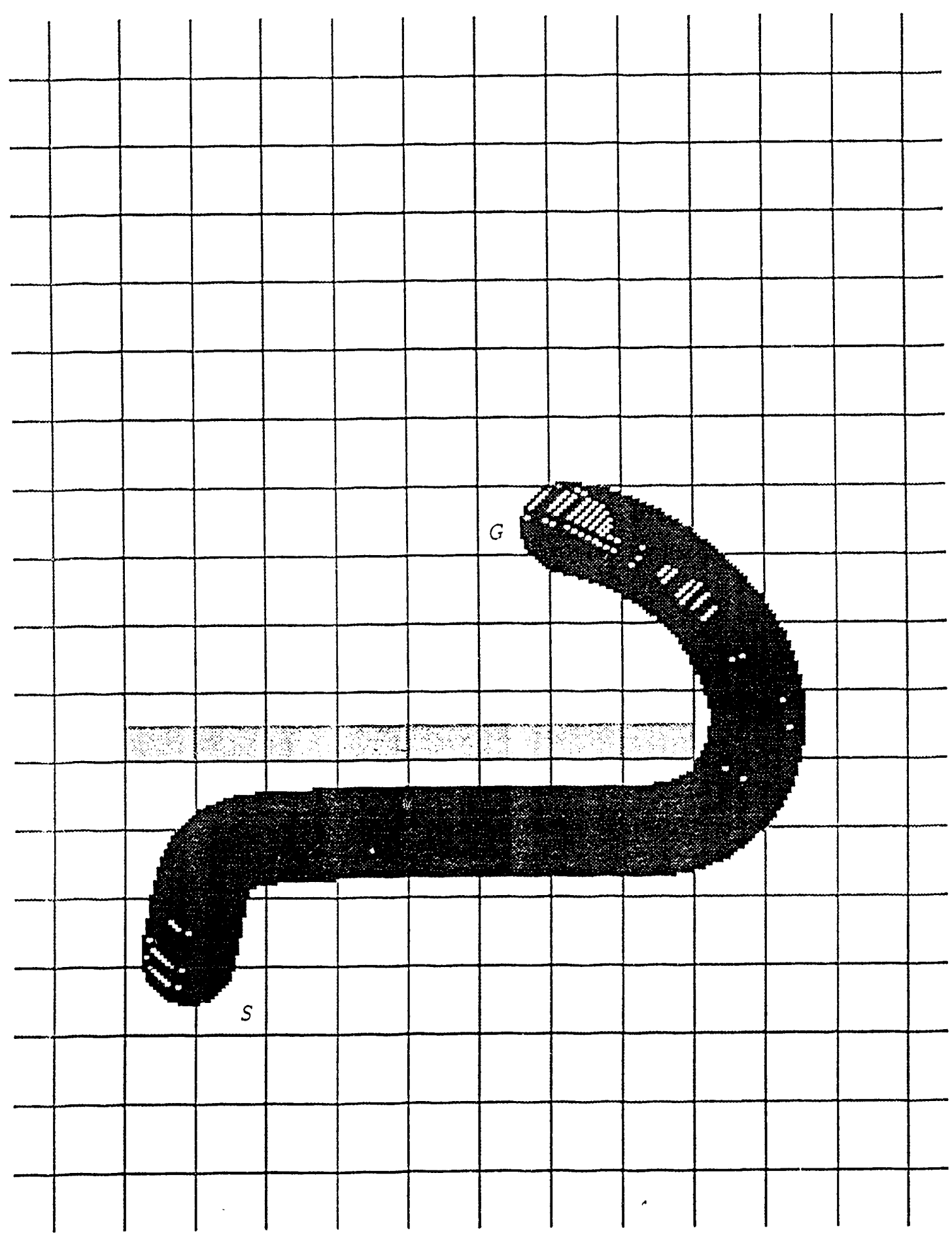

Fig. 5. Sample run of the platform illustrating basic obstacle avoidance, stable wall following, and no "trapping" in local minimia. $S$ and $G$ denote the start and goal locations. 


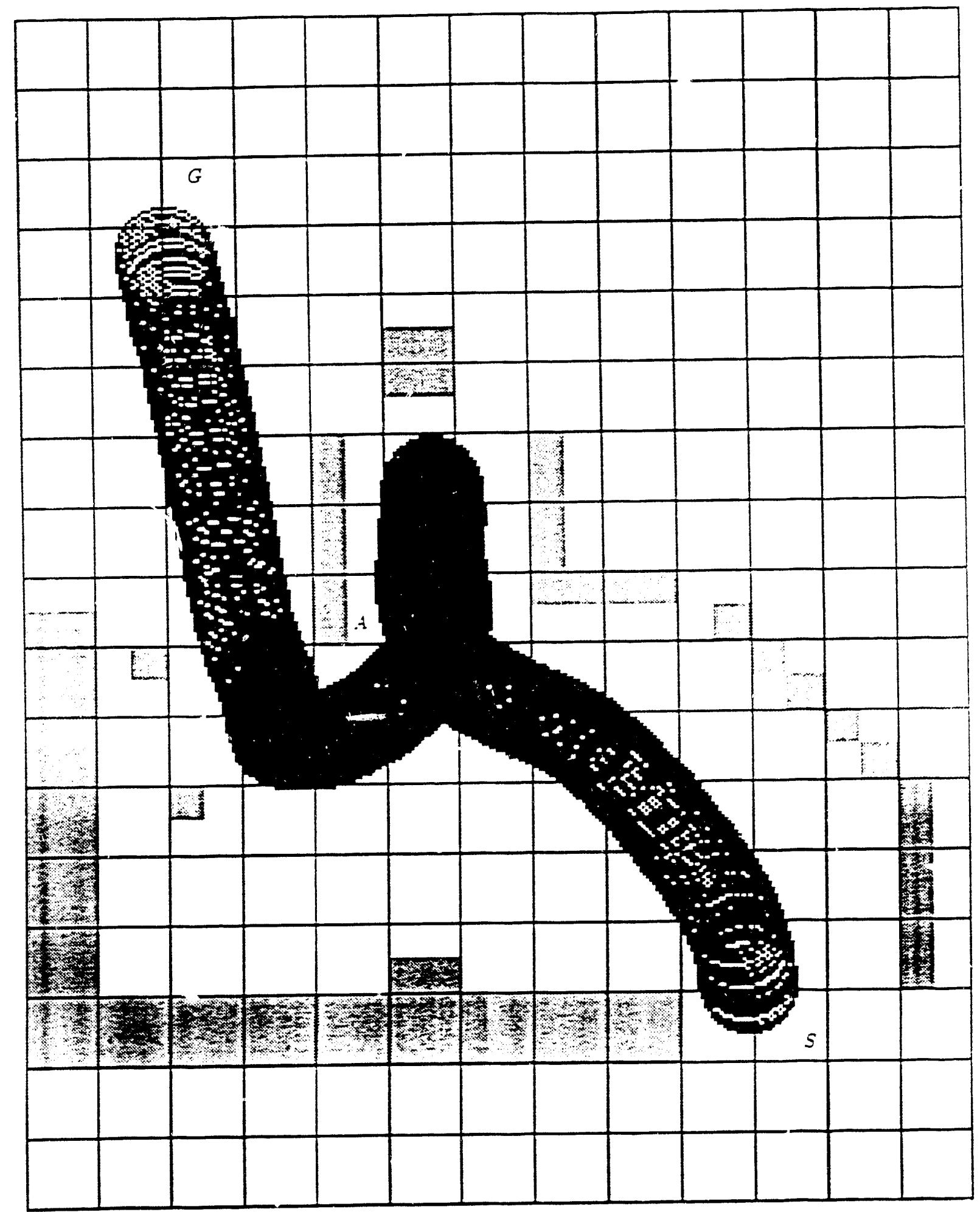

Fig. 6. Sample run of the platform illustrating obstacle avoidance in more complex environments, motion in corridors, and no "trapping" in local minimum at end of blocked corridor. 


\section{REFERENCES}

1. "Uncertainty in Artificial Intelligence," eds. L. N. Kanal and J. F. Lemmer, NorthHolland, New Vork (1988).

2. L. A. Zadeh, "Fuzzy Set," Information and Control $\underline{8}, 338-353$ (1965).

3. L. A. Zadeh, "Outline of a New Approach to the Analysis of Complex Systems and Decision-Making Approach," IEEE Transactions on Systems, Man, and Cybernetics SME-3(1), 28-45 (January 1973).

4. L. A. Zadeh, "Fuzzy Logic," IEEE Computer 21(4), 83-93 (April 1988).

5. "Fuzzy Sets and Their Applications to Cognitive and Decision Processes," eds. L. A. Zadeh, K. S. Fu, K. Tanaka, and M. Shinmra, Academic Press, Inc., New York (1975).

6. S. Yasunobu, S. Miyamoto, T. Takaoka, and H. Ohsihima, "Application of Predictive Fuzzy Control to Automatic Train Operation Controller," in Proc. IECON :84 (1984), pp. 657-662.

7. L. P. Holmblad and J. J. Ostergaard, "Control of a Cement Kiln by Fuzzy Logic," Fuzzy Information and Decision Processes, eds. M. M. Gupta and E. Ssnchez, 359-399 (1982).

8. L. I. Larkin, "A Fuzzy Logic Controller for Aircraft Flight Control," Industrial Applications of Fuzzy Control, ed. M. Sugeno, 87-103 (1985).

9. H. Ono, T. Ohnishi, and Y. Terada, "Combustion Control of Refuse Incineration Plant by Fuzzy Logic," in Proc. 2nd Inter. Fuzzy Systems Association Congress (July 198T), pp. 345-348.

10. M. Sugeno, et al., "Fuzzy Algorithmic Control of Model Car by Oral Instructions;" Fuzzy Sets and Systems 32, 207-219 (1989).

11. T. Yamakawa, "Stabilization of an Inverted Pendulum by a High-Speed Fuzzy Logic Controller Hardware System," Fuzzy Sets and Systems 32, 161-180 (1989).

12. K. A. Hirota and S. Hachisu, "Fuzzy Controlled Robot Arm Playing Two Dimensional Ping-Pong Game," Fuzzy Sets and Systems 32, 149-159 (1989).

13. H. Watanabe, W. Dettloff, and E. Yount, "A VLSI Fuzzy Logic Inference Engine for

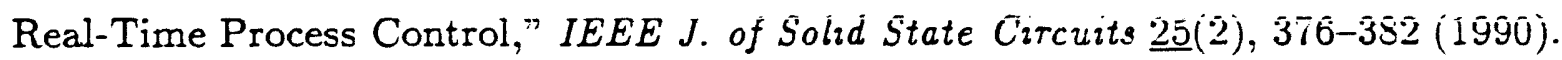


14. J. R. Symon and H. Watanabe, "Single Board System for Fuzzy Inference," in Proc. Workshop on Software Tools for Distributed Inteliigent Control Systems (September 1990), pp. 253-261.

15. S. M. Killough arf F. G. Pin, "A Fully Omnidirectional Wheel Assembly for Robotic Vehicles," Trans. Am. Nucl. Soc. $\underline{61}$, 425-426 (1990).

16. S. M. Killough and F. G. Pin, "Design of an Omnidirectional and Holonomic Wheeled Platform Prototye," submitted to the 1992 IEEE Conference on Robotics and Automation, May 10-15, 1992, Nice, France.

17. R. A. Brooks, "Elephants Don't Play Chess," Robotics and Autonomous Systems $\underline{6}$ (12), 3-15 (1990).

17. R. C. Arkins, "Integrating Behavioral, Perceptual and World Knowledge in Reactive Navigation" Robotics and Autonomous Systems $\underline{6}$ (1-2), 105-122 (1990).

\section{DISCLAIMER}

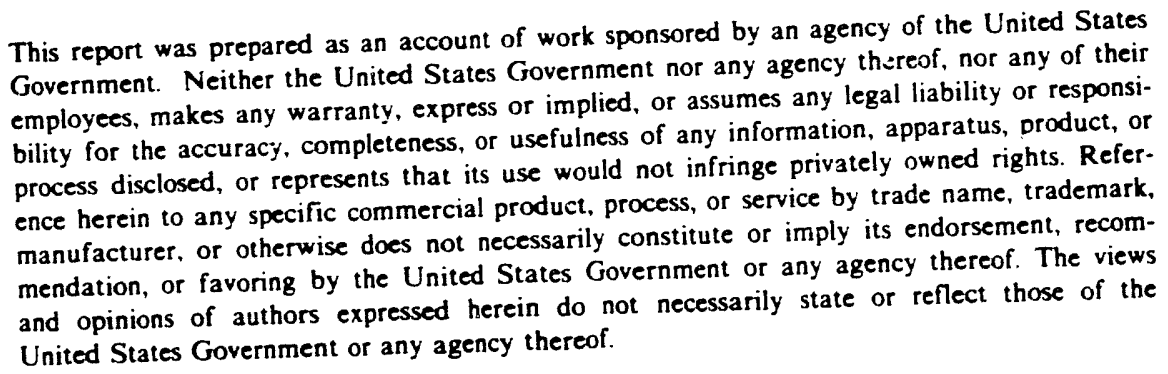



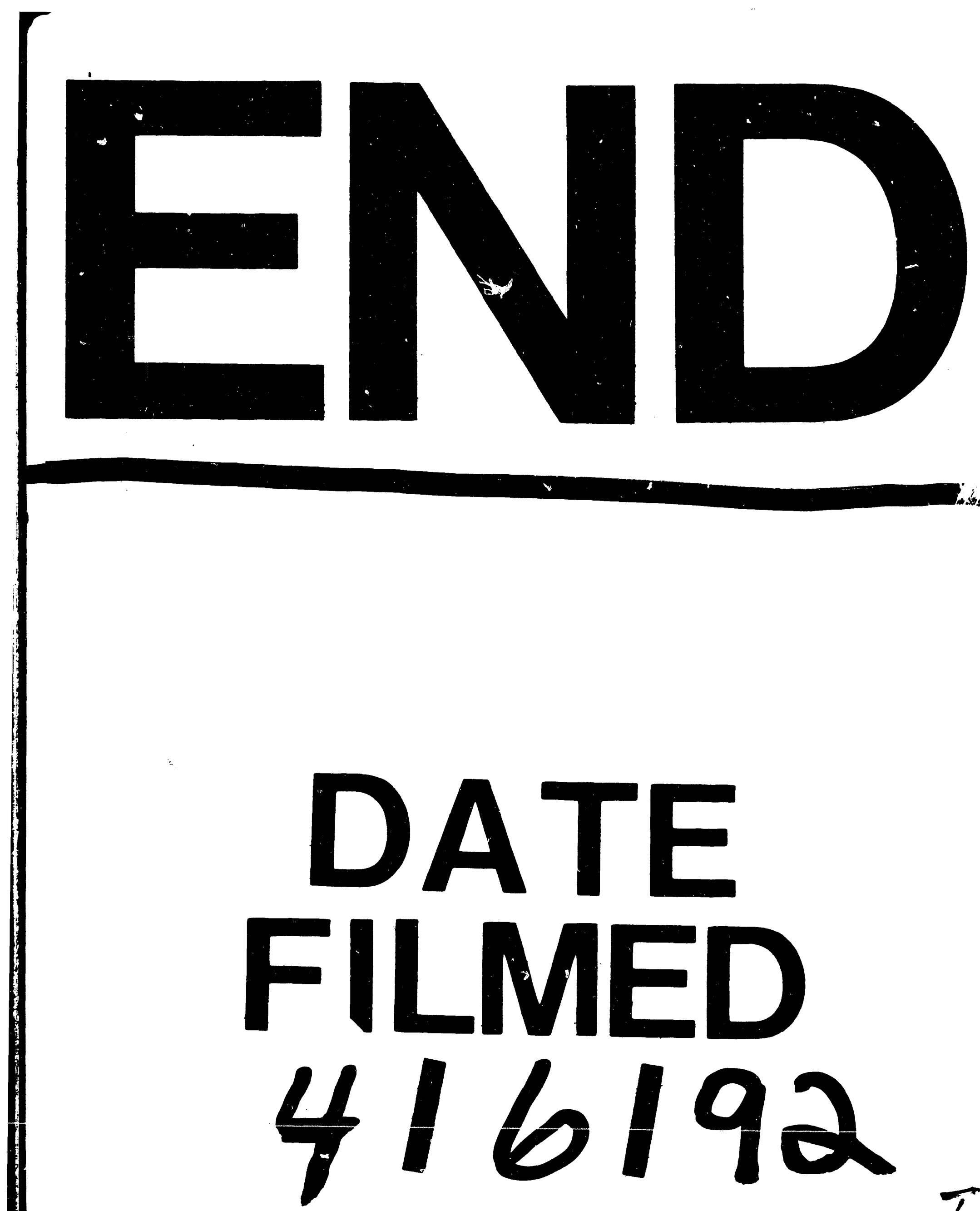

$I$ 
